\title{
ELLIPTIC CURVES WITH LARGE TATE-SHAFAREVICH GROUPS OVER A NUMBER FIELD
}

\author{
Kazuo Matsuno
}

\begin{abstract}
Let $p$ be a prime number and let $K$ be a cyclic Galois extension of $\mathbb{Q}$ of degree $p$. We prove that the $p$-rank of the Tate-Shafarevich group over $K$ of elliptic curves defined over $\mathbb{Q}$ can be arbitrarily large.
\end{abstract}

\section{Introduction}

For an elliptic curve $E$ defined over a number field $K$, the Tate-Shafarevich group $\amalg(E / K)$ of $E$ over $K$ is defined to be the abelian group consisting of the isomorphism classes of principal homogeneous spaces for $E$ over $K$ which are everywhere locally trivial. We have the following description of $\amalg(E / K)$ :

$$
\amalg(E / K)=\operatorname{Ker}\left(H^{1}(K, E(\bar{K})) \longrightarrow \prod_{v} H^{1}\left(K_{v}, E\left(\overline{K_{v}}\right)\right)\right) .
$$

Here $v$ runs over all primes of $K$. In this paper, we discuss the size of the TateShafarevich groups of elliptic curves over number fields. It is classically conjectured (but still unknown in general) that the Tate-Shafarevich group is finite for any elliptic curve over any number field of finite degree. Cassels, however, proved that there exists an elliptic curve defined over $\mathbb{Q}$ whose Tate-Shafarevich group has an arbitrarily large order. More precisely, Cassels [5] showed that the dimension over $\mathbb{F}_{3}$ of $\amalg(E / \mathbb{Q})[3]$, the 3-torsion subgroup of $\amalg(E / \mathbb{Q})$, is unbounded as $E$ varies over elliptic curves of $j$-invariant zero. After Cassels, the unboundedness of $\operatorname{dim}_{\mathbb{F}_{p}} \amalg(E / \mathbb{Q})[p]$ was studied by many authors and was proved for primes $p \leq 7$ or $p=13$. See the papers [1], [2], [11], [16], [18], [20], and some other papers cited in those.

It is not easy to prove the unboundedness of $\operatorname{dim}_{\mathbb{F}_{p}} \amalg(E / \mathbb{Q})[p]$ for an arbitrary $p$ by extending the method given in the above papers because many of them used the fact that there exist infinitely many elliptic curves over $\mathbb{Q}$ (with different $j$-invariants) which have isogenies of degree $p$. It is known that there exist only finitely many such elliptic curves for $p=11$ or $p \geq 17$. If we allow $K$ to vary over number fields of bounded degree and $E$ varies over elliptic curves over $K$, then the unboundedness of $\operatorname{dim}_{\mathbb{F}_{p}} \amalg(E / K)[p]$ has been proved for any $p$ by a similar method (cf. Kloosterman $[15])$. However, we cannot apply the same argument to showing the unboundedness for elliptic curves over a fixed number field $K$ when $p \geq 23$ since the modular curve $X_{0}(p)$ has genus greater than 1 and hence there exist only finitely many $K$-rational points on $X_{0}(p)$.

Received by the editors May 12, 2008.

2000 Mathematics Subject Classification. Primary 11G05. 
The aim of this paper is to prove that $\operatorname{dim}_{\mathbb{F}_{p}} \amalg(E / K)[p]$ is unbounded if $K$ is a fixed abelian field of degree $p$ and $E$ runs over elliptic curves over $\mathbb{Q}$. The main result is stated as follows.

Theorem A. Let $K$ be a Galois extension of $\mathbb{Q}$ such that $\operatorname{Gal}(K / \mathbb{Q}) \cong \mathbb{Z} / p \mathbb{Z}$ for a prime number $p$. Then, for any integer $k$, there exists an elliptic curve $E$ defined over $\mathbb{Q}$ satisfying $\operatorname{dim}_{\mathbb{F}_{p}} \amalg(E / K)[p] \geq k$.

More precisely, we will prove the unboundedness of the $n$-ranks of Tate-Shafarevich groups of elliptic curves over a fixed cyclic extension of $\mathbb{Q}$ of degree $n$, where $n$ is a positive integer not divisible by 4 (Theorems 5.1). We remark that the assertion of Theorem A does not follow immediately from the unboundedness of $\operatorname{dim}_{\mathbb{F}_{p}} \amalg(E / \mathbb{Q})[p]$, which is known in the case $p \leq 7$ or $p=13$. Indeed, the natural map $\amalg(E / \mathbb{Q}) \rightarrow$ $\amalg(E / K)$ might have a large kernel of exponent $p$ if the degree of $K$ is divisible by $p$.

Our proof of Theorem A is separated into two steps. The first step is to give a lower bound for the size of the $p$-Selmer $\operatorname{group} \operatorname{Sel}_{p}(E / K)$ of an elliptic curve $E$ over $K$. In order to obtain a nontrivial lower bound, we investigate the difference of Selmer groups in the cyclic Galois extension $K / \mathbb{Q}$ of degree $p$. In [21], Mazur studied the behavior of the $p^{\infty}$-Selmer groups of abelian varieties in an infinite Galois extension with Galois group isomorphic to $\mathbb{Z}_{p}$ and proved a result which is often called "Mazur's control theorem" (cf. [12, Section 1]). We apply a similar argument to our situation (Proposition 3.2). The main ingredient of the proof is the Cassels-Poitou-Tate global duality.

This lower bound enables us to show that $\operatorname{dim}_{\mathbb{F}_{p}} \operatorname{Sel}_{p}(E / K)$ is unbounded as $E$ varies over elliptic curves defined over $\mathbb{Q}$ (Corollary 4.4). This implies the unboundedness of either $\operatorname{rank}_{\mathbb{Z}} E(K)$ or $\operatorname{dim}_{\mathbb{F}_{p}} \amalg(E / K)[p]$ (see the exact sequence (1) in Section 2). The second step of the proof of Theorem A is to construct an elliptic curve $E$ with large $p$-Selmer group and with small Mordell-Weil group over $K$. For an odd $p$, we will construct an elliptic curve $E$ such that $\operatorname{Sel}_{p}(E / K)$ is arbitrarily large and $\operatorname{Sel}_{2}(E / K)$ is small (bounded by some constant) by using Kramer's argument in [18] and a result coming from sieve methods. For $p=2$, the upper bound of the MordellWeil rank is obtained by a result of Hoffstein-Luo [14] on the existence of a quadratic twist of an elliptic curve such that the central value of the Hasse-Weil $L$-function is nonzero and the conductor has only a few prime factors. The proofs are given in Section 5 for odd $p$ and in Section 6 for $p=2$.

Kloosterman's result [15] mentioned above is the unboundedness of $\operatorname{dim}_{\mathbb{F}_{p}}$ $\amalg(E / K)[p]$ as both $K$ and $E$ vary. Our main result, Theorem A, improves this by fixing the base field $K$. (We remark that the degree of $K$ in Theorem $\mathrm{A},[K: \mathbb{Q}]=p$, is smaller than that considered in [15].) Recently, Clark and Sharif gave in [7] a different improvement of Kloosterman's result that $\operatorname{dim}_{\mathbb{F}_{p}} \amalg(E / K)[p]$ is unbounded for any fixed elliptic curve $E$ over $\mathbb{Q}$ as $K$ varies over number fields of degree $p$ (not necessarily Galois over $\mathbb{Q}$ ). We will give another proof of their result for $p=2$ (see the end of Section 4).

Proposition B. Let $E$ be an elliptic curve defined over $\mathbb{Q}$. Then, for any integer $k$, there exists a quadratic field $K$ satisfying $\operatorname{dim}_{\mathbb{F}_{2}} \amalg(E / K)[2] \geq k$. 


\section{Notation}

For an abelian group $M$ and a positive integer $n$, we denote by $M[n]$ the subgroup of $M$ annihilated by $n$. If $M$ is a torsion abelian group, then we denote by $M^{(p)}$ the $p$-primary component of $M$ for each prime $p$, i.e., $M^{(p)}:=\cup_{m} M\left[p^{m}\right]$. For a finite abelian group $M$, we denote by $\operatorname{rk}_{n} M$ the largest integer $k$ such that $M$ contains a subgroup isomorphic to $(\mathbb{Z} / n \mathbb{Z})^{\oplus k}$. By definition, we have $\operatorname{rk}_{n} M=\operatorname{rk}_{n}(M[n])$ in any case, and $\operatorname{rk}_{p} M=\operatorname{dim}_{\mathbb{F}_{p}} M$ if $p M=0$ for a prime $p$.

For an elliptic curve $E$ defined over a number field $K$, we put $E[n]:=E(\bar{K})[n]$. Then the $n$-Selmer group $\operatorname{Sel}_{n}(E / K)$ of $E$ over $K$ is defined as follows:

$$
\operatorname{Sel}_{n}(E / K):=\operatorname{Ker}\left(H^{1}(K, E[n]) \longrightarrow \prod_{v} H^{1}\left(K_{v}, E\left(\overline{K_{v}}\right)\right)\right)
$$

where $v$ runs over all primes of $K$. By definition, we have an exact sequence

$$
0 \longrightarrow E(K) / n E(K) \longrightarrow \operatorname{Sel}_{n}(E / K) \longrightarrow \amalg(E / K)[n] \longrightarrow 0 .
$$

For a prime number $p$, we denote by $\operatorname{Sel}_{p^{\infty}}(E / K)$ the inductive limit of $\operatorname{Sel}_{p^{m}}(E / K)$ under the maps induced by the natural inclusions $E\left[p^{m}\right] \hookrightarrow E\left[p^{m+1}\right]$. We have

$$
\operatorname{Sel}_{p \infty}(E / K)=\operatorname{Ker}\left(H^{1}\left(K, E\left[p^{\infty}\right]\right) \longrightarrow \prod_{v} H^{1}\left(K_{v}, E\left(\overline{K_{v}}\right)\right)\right)
$$

where $E\left[p^{\infty}\right]=\cup_{m} E\left[p^{m}\right]$ is the group of all $p$-power torsion points of $E$.

\section{Consequences of global duality}

In this section, we recall some facts obtained from the global duality. We assume that $E$ is an elliptic curve defined over $\mathbb{Q}$.

Proposition 3.1. Let $p$ be a prime number and $S$ a finite set of primes of $\mathbb{Q}$ containing $p$, the unique archimedean prime, and all bad reduction primes for $E$. Then $\operatorname{Sel}_{p}(E / \mathbb{Q})$ coincides with the kernel of the map

$$
\varphi: H^{1}\left(\mathbb{Q}_{S} / \mathbb{Q}, E\left[p^{\infty}\right]\right) \longrightarrow \prod_{v \in S} H^{1}\left(\mathbb{Q}_{v}, E\left(\overline{\mathbb{Q}_{v}}\right)\right)^{(p)},
$$

where $\mathbb{Q}_{S}$ denotes the maximal extension of $\mathbb{Q}$ unramified outside $S$. Furthermore, we have

$$
\operatorname{rk}_{p} \operatorname{Coker}(\varphi)[p] \leq \operatorname{rank}_{\mathbb{Z}_{p}} \operatorname{Sel}_{p^{\infty}}(E / \mathbb{Q})^{\vee}+\operatorname{rk}_{p} E(\mathbb{Q})[p],
$$

where $\operatorname{Sel}_{p^{\infty}}(E / \mathbb{Q})^{\vee}$ is the Pontryagin dual of $\operatorname{Sel}_{p^{\infty}}(E / \mathbb{Q})$.

Remark. We have $\operatorname{rank}_{\mathbb{Z}_{p}} \operatorname{Sel}_{p^{\infty}}(E / \mathbb{Q})^{\vee}=\operatorname{rank}_{\mathbb{Z}} E(\mathbb{Q})$ if $\amalg(E / \mathbb{Q})^{(p)}$ is finite.

Proof. The first assertion is well-known (cf. [22, Corollary I.6.6]). The second assertion follows immediately from [8, (4) and Lemma 1.8].

Let $K$ be a cyclic Galois extension of $\mathbb{Q}$ of finite degree. For a (non-archimedean or archimedean) prime $v$ of $\mathbb{Q}$, we define $W_{v, K}$ by

$$
W_{v, K}:=\operatorname{Ker}\left(H^{1}\left(\mathbb{Q}_{v}, E\left(\overline{\mathbb{Q}_{v}}\right)\right) \longrightarrow H^{1}\left(K_{w}, E\left(\overline{\mathbb{Q}}_{v}\right)\right)\right),
$$

where $w$ is a prime of $K$ lying above $v$. The definition of $W_{v, K}$ is independent of the choice of $w$. It is known that $W_{v, K}$ is finite. 
Proposition 3.2. Let $K / \mathbb{Q}$ be a cyclic Galois extension with Galois group $G=$ $\operatorname{Gal}(K / \mathbb{Q})$. Suppose that the set $S$ in the statement of Proposition 3.1 contains the primes ramified in $K / \mathbb{Q}$. Then $\operatorname{Sel}_{p}(E / K)$ contains a subgroup $\mathcal{M}$ which sits in the following exact sequence:

$$
0 \longrightarrow X \longrightarrow \operatorname{Sel}_{p^{\infty}}(E / \mathbb{Q}) \longrightarrow \mathcal{M} \longrightarrow\left(\prod_{v \in S} W_{v, K}^{(p)}\right) / X^{\prime} \longrightarrow Y \longrightarrow 0 .
$$

Here $X, X^{\prime}$ and $Y$ are finite abelian p-groups satisfying

$$
\begin{aligned}
& \operatorname{rk}_{p} X, \operatorname{rk}_{p} X^{\prime} \leq \operatorname{rk}_{p} E(\mathbb{Q})[p]+\delta, \\
& \operatorname{rk}_{p} Y \leq \operatorname{rank}_{\mathbb{Z}_{p}} \operatorname{Sel}_{p^{\infty}}(E / \mathbb{Q})^{\vee}+\operatorname{rk}_{p} E(\mathbb{Q})[p],
\end{aligned}
$$

where $\delta=1$ if $p=2$ and $\operatorname{rk}_{2} E(\mathbb{Q})[2]=1$, and $\delta=0$ if not.

Remark. The above $\mathcal{M}$ is of finite index in $\operatorname{Sel}_{p \infty}(E / K)^{G}$, the subgroup of $\operatorname{Sel}_{p^{\infty}}(E / K)$ consisting of $G$-invariant elements. Moreover, we have $\mathcal{M}=$ $\operatorname{Sel}_{p \infty}(E / K)^{G}$ if $E(\mathbb{Q})[p]=0$.

Proof. Let $\mathcal{M}^{\prime}$ be the image of the restriction map

$$
H^{1}\left(\mathbb{Q}_{S} / \mathbb{Q}, E\left[p^{\infty}\right]\right) \longrightarrow H^{1}\left(\mathbb{Q}_{S} / K, E\left[p^{\infty}\right]\right) .
$$

Then we have the commutative diagram

$$
\begin{array}{ccccccc}
0 \rightarrow & H^{1}\left(G, E(K)\left[p^{\infty}\right]\right) & \rightarrow & H^{1}\left(\mathbb{Q}_{S} / \mathbb{Q}, E\left[p^{\infty}\right]\right) & \rightarrow & \mathcal{M}^{\prime} \\
& \downarrow \psi & & & \downarrow \varphi & \downarrow \varphi_{K} \\
0 \rightarrow & \prod_{v \in S} W_{v, K}^{(p)} & \rightarrow & \prod_{v \in S} H^{1}\left(\mathbb{Q}_{v}, E\left(\overline{\mathbb{Q}_{v}}\right)\right)^{(p)} & \rightarrow & \prod_{v \in S} \prod_{w \mid v} H^{1}\left(K_{w}, E\left(\overline{\mathbb{Q}_{v}}\right)\right)^{(p)} & \rightarrow 0
\end{array} \rightarrow
$$

with exact rows. Put $\mathcal{M}=\operatorname{Ker}\left(\varphi_{K}\right), X=\operatorname{Ker}(\psi)$ and $X^{\prime}=\operatorname{Im}(\psi)$, where $\varphi_{K}$ and $\psi$ are the vertical maps in the above diagram. By definition, $\mathcal{M}$ is contained in $\operatorname{Sel}_{p \infty}(E / K)$, and we have an exact sequence

$$
0 \longrightarrow X \longrightarrow \operatorname{Sel}_{p^{\infty}}(E / \mathbb{Q}) \longrightarrow \mathcal{M} \longrightarrow\left(\prod_{v \in S} W_{v, K}^{(p)}\right) / X^{\prime} \longrightarrow \operatorname{Coker}(\varphi)
$$

by the snake lemma. By putting $Y$ as the image of the last map of this sequence, we obtain the exact sequence (2). The assertion on $\operatorname{rk}_{p} Y$ follows immediately from Proposition 3.1. Since we have $\operatorname{rk}_{p} X, \operatorname{rk}_{p} X^{\prime} \leq \operatorname{rk}_{p} H^{1}\left(G, E(K)\left[p^{\infty}\right]\right)$ by definition, the proof of this proposition is reduced to showing

$$
\operatorname{rk}_{p} H^{1}\left(G, E(K)\left[p^{\infty}\right]\right) \leq \operatorname{rk}_{p} E(\mathbb{Q})[p]+\delta .
$$

Let $K^{\prime}$ be the maximal $p$-extension of $\mathbb{Q}$ contained in $K$ and fix a generator $\sigma$ of $G^{\prime}=\operatorname{Gal}\left(K^{\prime} / \mathbb{Q}\right)$. Since $G^{\prime}$ is cyclic, we have

$$
H^{1}\left(G, E(K)\left[p^{\infty}\right]\right) \cong H^{1}\left(G^{\prime}, E\left(K^{\prime}\right)\left[p^{\infty}\right]\right) \cong \operatorname{Ker}\left(N_{K^{\prime} / \mathbb{Q}}\right) /(\sigma-1)\left(E\left(K^{\prime}\right)\left[p^{\infty}\right]\right),
$$

where $N_{K^{\prime} / \mathbb{Q}}: E\left(K^{\prime}\right)\left[p^{\infty}\right] \rightarrow E(\mathbb{Q})\left[p^{\infty}\right]$ is the norm map. In particular, we have

$$
\mathrm{rk}_{p} H^{1}\left(G, E(K)\left[p^{\infty}\right]\right) \leq \mathrm{rk}_{p} E\left(K^{\prime}\right)\left[p^{\infty}\right]=\operatorname{rk}_{p} E\left(K^{\prime}\right)[p] .
$$

Since $G^{\prime}$ is a $p$-group, $\operatorname{rk}_{p} E\left(K^{\prime}\right)[p]=0$ if and only if $\operatorname{rk}_{p} E(\mathbb{Q})[p]=0$. This implies $\operatorname{rk}_{2} E\left(K^{\prime}\right)[2] \leq \operatorname{rk}_{2} E(\mathbb{Q})[2]+\delta$ for $p=2$. If $p$ is odd, then $K^{\prime}$ contains no primitive $p$-th root of unity. Hence we have $\operatorname{rk}_{p} E\left(K^{\prime}\right)[p] \leq 1$ for any odd $p$, which implies $\operatorname{rk}_{p} E(\mathbb{Q})[p]=\operatorname{rk}_{p} E\left(K^{\prime}\right)[p]$. Thus we obtain the inequality (3) for any $p$. The proof has been completed. 
Remark. We cannot remove the term $\delta$ in (3). In fact, if we take $E$ as the elliptic curve defined by $y^{2}=(x-1)\left(x^{2}+x-1\right)$, the curve 40A3 in [9], and take $K$ as the cyclotomic field of conductor 5 , then we have $E(\mathbb{Q})\left[2^{\infty}\right] \cong \mathbb{Z} / 4 \mathbb{Z}$ and $E(K)\left[2^{\infty}\right]=$ $E(\mathbb{Q}(\sqrt{5}))\left[2^{\infty}\right] \cong \mathbb{Z} / 4 \mathbb{Z} \oplus \mathbb{Z} / 2 \mathbb{Z}$. One sees that the norm map $N_{K / \mathbb{Q}}$ is the zero map and $(\sigma-1)\left(E(K)\left[2^{\infty}\right]\right)=2 E(\mathbb{Q})\left[2^{\infty}\right]$, where $\sigma$ is a generator of $G=\operatorname{Gal}(K / \mathbb{Q})$. Therefore, $H^{1}\left(G, E(K)\left[2^{\infty}\right]\right) \cong \operatorname{Ker}\left(N_{K / \mathbb{Q}}\right) /(\sigma-1)\left(E(K)\left[2^{\infty}\right]\right) \cong(\mathbb{Z} / 2 \mathbb{Z})^{\oplus 2}$, which implies $\operatorname{rk}_{2} H^{1}\left(G, E(K)\left[2^{\infty}\right]\right)=2=\operatorname{rk}_{2} E(\mathbb{Q})[2]+1$.

Corollary 3.3. For any prime number $p$ and any positive integer e, we have

$$
\operatorname{rk}_{p^{e}} \operatorname{Sel}_{p^{e}}(E / K) \geq \sum_{v \in S} \operatorname{rk}_{p^{e}} W_{v, K}-2 \operatorname{rk}_{p} E(\mathbb{Q})[p]-\delta,
$$

where $\delta$ and $S$ are as in Proposition 3.2.

Proof. Let $\mathcal{M}, X^{\prime}$ and $Y$ be as in Proposition 3.2. Put $r=\operatorname{rank}_{\mathbb{Z}_{p}} \operatorname{Sel}_{p^{\infty}}(E / \mathbb{Q})^{\vee}$ and $t=\operatorname{rk}_{p} E(\mathbb{Q})[p]$. By the exact sequence (2) in Proposition 3.2, the maximal divisible subgroup $\mathcal{D}$ of $\mathcal{M}$ is isomorphic to $\left(\mathbb{Q}_{p} / \mathbb{Z}_{p}\right)^{\oplus r}$ and we have an exact sequence of finite abelian $p$-groups:

$$
\mathcal{M} / \mathcal{D} \longrightarrow\left(\prod_{v \in S} W_{v, K}^{(p)}\right) / X^{\prime} \longrightarrow Y \longrightarrow 0
$$

Although the $p^{e}$-rank is not "additive" for short exact sequences in general, the above sequence implies the inequality

$$
\operatorname{rk}_{p^{e}} \mathcal{M} / \mathcal{D} \geq \sum_{v \in S} \operatorname{rk}_{p^{e}} W_{v, K}^{(p)}-\operatorname{rk}_{p} X^{\prime}-\operatorname{rk}_{p} Y
$$

Therefore, as an abelian group, $\mathcal{M}$ is isomorphic to the direct sum of $\left(\mathbb{Q}_{p} / \mathbb{Z}_{p}\right)^{\oplus r}$ and a finite abelian $p$-group whose $p^{e}$-rank is not less than $\sum_{v \in S} \operatorname{rk}_{p^{e}} W_{v, K}^{(p)}-r-2 t-\delta$. Since $\mathcal{M}$ is a subgroup of $\operatorname{Sel}_{p^{\infty}}(E / K)$ and there exists a surjection $\operatorname{Sel}_{p^{e}}(E / K) \rightarrow$ $\operatorname{Sel}_{p \infty}(E / K)\left[p^{e}\right]$, we have

$$
\begin{aligned}
\operatorname{rk}_{p^{e}} \operatorname{Sel}_{p^{e}}(E / K) & \geq \operatorname{rk}_{p^{e}} \mathcal{M}\left[p^{e}\right] \\
& \geq r+\sum_{v \in S} \operatorname{rk}_{p^{e}} W_{v, K}^{(p)}-r-2 t-\delta \\
& =\sum_{v \in S} \operatorname{rk}_{p^{e}} W_{v, K}-2 t-\delta .
\end{aligned}
$$

The proof has been completed.

Remark. In the case $e=1$, one can improve the assertion of the above corollary as

$$
\operatorname{rk}_{p} \operatorname{Sel}_{p}(E / K) \geq \sum_{v \in S} \operatorname{rk}_{p} W_{v, K}-\operatorname{rk}_{p} E(\mathbb{Q})[p]
$$

by using the fact that the kernel of $\operatorname{Sel}_{p}(E / K) \rightarrow \operatorname{Sel}_{p}(E / K)[p]$ is isomorphic (as an abelian group) to $E(K)[p]$. 


\section{Large Selmer groups}

In this section, we give some sufficient conditions for $W_{\ell, K}$ to be nontrivial. By Corollary 3.3, this enables us to construct elliptic curves defined over $\mathbb{Q}$ which have large Selmer groups over $K$. We keep the assumptions that the elliptic curve $E$ is defined over $\mathbb{Q}$ and $K$ is a cyclic Galois extension of $\mathbb{Q}$.

Lemma 4.1. Let $\ell$ be a prime number satisfying the following conditions for a positive integer $n$ prime to $\ell$.

(i) $E$ has split multiplicative reduction at $\ell$.

(ii) The inertia degree of $\ell$ in $K / \mathbb{Q}$ is divisible by $n$.

(iii) The Tamagawa factor $c_{\ell}$ of $E$ at $\ell$ is divisible by $n$.

Then $W_{\ell, K}$ contains a subgroup isomorphic to $\mathbb{Z} / n \mathbb{Z}$, i.e., $\mathrm{rk}_{n} W_{\ell, K} \geq 1$.

Proof. Fix a prime $\mathfrak{l}$ of $K$ lying above $\ell$. Let $L$ be the maximal unramified extension of $\mathbb{Q}_{\ell}$ in $K_{\mathfrak{l}}$ and put $G^{\prime}=\operatorname{Gal}\left(L / \mathbb{Q}_{\ell}\right)$. Then we have an injection $H^{1}\left(G^{\prime}, E(L)\right) \hookrightarrow W_{\ell, K}$ by the inflation-restriction sequence. Hence it suffices to show that $H^{1}\left(G^{\prime}, E(L)\right)$ has an element of order $n$. If we denote by $E_{0}(L)$ the subgroup of $E(L)$ consisting of the points with non-singular reduction, then we have

$$
H^{1}\left(G^{\prime}, E(L)\right) \cong H^{1}\left(G^{\prime}, E(L) / E_{0}(L)\right)
$$

(cf. [21, Proposition 4.3]). By the assumption (i) and the fact that $L / \mathbb{Q}_{\ell}$ is unramified, $E(L) / E_{0}(L)$ is a cyclic group of order $c_{\ell}$ and $G^{\prime}$ acts trivially on it. Hence we have

$$
H^{1}\left(G^{\prime}, E(L)\right) \cong \operatorname{Hom}\left(G^{\prime}, E(L) / E_{0}(L)\right) \cong \mathbb{Z} / g \mathbb{Z},
$$

where $g$ is the greatest common divisor of $c_{\ell}$ and the order of $G^{\prime}$. By (ii) and (iii), $g$ is divisible by $n$. Thus the claim has been proved.

Lemma 4.2. Let $\ell$ be a prime number satisfying the following conditions for a positive integer $n$ prime to $\ell$.

(i) $E$ has good reduction at $\ell$.

(ii) The ramification index of $\ell$ in $K / \mathbb{Q}$ is divisible by $n$.

(iii) $E\left(\mathbb{Q}_{\ell}\right)$ contains an element of order $n$.

Then $W_{\ell, K}$ contains a subgroup isomorphic to $\mathbb{Z} / n \mathbb{Z}$.

Proof. Since we have an isomorphism $E\left(\mathbb{Q}_{\ell}\right) / n E\left(\mathbb{Q}_{\ell}\right) \stackrel{\sim}{\longrightarrow} H^{1}\left(\mathbb{Q}_{\ell}, E\left(\overline{\mathbb{Q}_{\ell}}\right)\right)[n]$ by the Tate local duality (cf. [22, Corollary I.3.4]), there exists an element $\alpha \in H^{1}\left(\mathbb{Q}_{\ell}, E\left(\overline{\mathbb{Q}_{\ell}}\right)\right)$ of order $n$ by the assumption (iii). By [19, Corollary 1], $\alpha$ becomes trivial over $K_{\mathfrak{r}}$ under the assumptions (i) and (ii), i.e., $\alpha \in W_{\ell, K}$. Thus, $W_{\ell, K}$ contains an element of order $n$, as desired.

By these lemmas, we obtain a lower bound of Selmer groups.

Definition. For a cyclic Galois extension $K$ over $\mathbb{Q}$ of degree $n$, let $T_{E, K}$ be the set of prime numbers $\ell \nmid n$ satisfying the assumptions either of Lemmas 4.1 or 4.2. Denote by $t_{E, K}$ the cardinality of $T_{E, K}$.

Proposition 4.3. Let $K$ be a cyclic Galois extension of $\mathbb{Q}$ of degree $n$. Then we have

$$
\operatorname{rk}_{n} \operatorname{Sel}_{n}(E / K) \geq t_{E, K}-2 \max \left\{\operatorname{rk}_{p} E(\mathbb{Q})[p]|p| n\right\}-\delta^{\prime} \geq t_{E, K}-4,
$$

where $\delta^{\prime}=1$ if $n$ is even and $\operatorname{rk}_{2} E(\mathbb{Q})[2]=1$, and $\delta^{\prime}=0$ if not. 
Proof. By Lemmas 4.1 and 4.2 , we have $\operatorname{rk}_{n}\left(W_{\ell, K}\right) \geq 1$ for any $\ell \in T_{E, K}$. Hence the assertion follows immediately from Corollary 3.3.

By using this lower bound, we have the following results on the unboundedness of $p$-Selmer groups.

Corollary 4.4. Let $p$ be a prime number. Then, for any cyclic Galois extension $K / \mathbb{Q}$ of degree $p$, we have

$$
\sup \left\{\operatorname{dim}_{\mathbb{F}_{p}} \operatorname{Sel}_{p}(E / K) \mid E \text { is defined over } \mathbb{Q}\right\}=+\infty .
$$

Proof. For any positive integer $k$, take prime numbers $\ell_{1}, \cdots, \ell_{k}$ not equal to $p$ which remain primes in $K$. Then there exists an elliptic curve $E^{\prime}$ defined over $\mathbb{Q}$ whose $j$-invariant is equal to $\left(\ell_{1} \cdots \ell_{k}\right)^{-p}$. We can take a quadratic twist $E$ of $E^{\prime}$ such that $E$ has split multiplicative reduction at each $\ell_{i}$. Since $\operatorname{ord}_{\ell_{i}}\left(j_{E}\right)=\operatorname{ord}_{\ell_{i}}\left(j_{E^{\prime}}\right)=-p$, the Tamagawa factor of $E$ at $\ell_{i}$ is equal to $p$. Therefore, the primes $\ell_{1}, \cdots, \ell_{k}$ satisfy the conditions of Lemma 4.1, i.e., $\ell_{1}, \cdots, \ell_{k} \in T_{E, K}$. By Proposition 4.3, we have $\operatorname{dim}_{\mathbb{F}_{p}} \operatorname{Sel}_{p}(E / K) \geq k-4$, which implies the assertion of this corollary.

Corollary 4.5. Let $p$ be a prime number. For any elliptic curve $E$ defined over $\mathbb{Q}$, we have

$$
\sup \left\{\operatorname{dim}_{\mathbb{F}_{p}} \operatorname{Sel}_{p}(E / K) \mid K / \mathbb{Q} \text { is a cyclic extension of degree } p\right\}=+\infty .
$$

Proof. There exist infinitely many odd prime numbers which split completely in the extension $\mathbb{Q}(E[p]) / \mathbb{Q}$. For any positive integer $k$, take such primes $\ell_{1}, \cdots, \ell_{k}$ at which $E$ has good reduction. Then $E[p]$ is contained in $E\left(\mathbb{Q}_{\ell_{i}}\right)$ for each $i$. By a property of the Weil pairing, $\mathbb{Q}_{\ell_{i}}^{\times}$contains a primitive $p$-th root of unity, i.e., $\ell_{i} \equiv 1(\bmod p)$. Hence there exists an abelian field $K$ of degree $p$ and of conductor $\ell_{1} \cdots \ell_{k}$. Then the primes $\ell_{1}, \cdots, \ell_{k}$ satisfy the conditions of Lemma 4.2 , i.e., $\ell_{1}, \cdots, \ell_{k} \in T_{E, K}$. Thus, we have $\operatorname{dim}_{\mathbb{F}_{p}} \operatorname{Sel}_{p}(E / K) \geq k-4$ by Proposition 4.3. This implies the assertion.

We conclude this section by giving a proof of Proposition B in the introduction. Let $E$ be an elliptic curve defined over $\mathbb{Q}$ with conductor $N$. As in the proof of Corollary 4.5, take odd prime numbers $\ell_{1}, \cdots, \ell_{k} \nmid N$ which split completely in the Galois extension $\mathbb{Q}(E[2]) / \mathbb{Q}$. By results of Waldspurger (cf. [4, Theorem in Section 0]) and Kolyvagin $([17])$, there exists a quadratic field $K$ such that all $\ell_{1}, \cdots, \ell_{k}$ ramify in $K / \mathbb{Q}$ and $\operatorname{rank}_{\mathbb{Z}} E^{\prime}(\mathbb{Q})=0$, where $E^{\prime}$ is the quadratic twist of $E$ corresponding to $K$. Then we have $\operatorname{rank}_{\mathbb{Z}} E(K)=\operatorname{rank}_{\mathbb{Z}} E(\mathbb{Q})+\operatorname{rank}_{\mathbb{Z}} E^{\prime}(\mathbb{Q})=\operatorname{rank}_{\mathbb{Z}} E(\mathbb{Q})$. By Corollary 3.3 and Lemma 4.2 , we have $\operatorname{dim}_{\mathbb{F}_{2}} \operatorname{Sel}_{2}(E / K) \geq k-4$ and

$$
\operatorname{dim}_{\mathbb{F}_{2}} \amalg(E / K)[2] \geq \operatorname{dim}_{\mathbb{F}_{2}} \operatorname{Sel}_{2}(E / K)-\operatorname{rank}_{\mathbb{Z}} E(K)-2 \geq k-6-\operatorname{rank}_{\mathbb{Z}} E(\mathbb{Q}) .
$$

Since $\operatorname{rank}_{\mathbb{Z}} E(\mathbb{Q})$ is independent of $k$, this completes the proof of Proposition B by taking $k$ arbitrarily large.

\section{Large Tate-Shafarevich groups}

In this section, we prove the following result, which implies the statement of Theorem $\mathrm{A}$ in the introduction for odd primes $p$. 
Theorem 5.1. Let $K$ be a cyclic Galois extension of $\mathbb{Q}$ of odd degree n. Then, for any positive integer $\kappa$, there exists an elliptic curve $E$ defined over $\mathbb{Q}$ such that $\amalg(E / K)$ contains a subgroup isomorphic to $(\mathbb{Z} / 2 n \mathbb{Z})^{\oplus \kappa}$, i.e., $\operatorname{rk}_{2 n} \amalg(E / K)[2 n] \geq \kappa$.

For a positive integer $k$, let $\ell_{1}, \cdots, \ell_{k}, m_{1}, \cdots, m_{k}$ be distinct odd prime numbers satisfying the following conditions:

(A1) $\ell_{i} \equiv 1(\bmod 4)$ and $\ell_{i} \nmid n$ for any $i$.

(A2) $m_{j} \nmid n$ for any $j$.

(A3) All $\ell_{1}, \cdots, \ell_{k}, m_{1}, \cdots, m_{k}$ remain prime in $K$.

(A4) $\left(\frac{m_{j}}{\ell_{i}}\right)=(-1)^{\delta_{i, j}}$ for any pair of $i$ and $j$, where $\delta_{i, j}$ is the Kronecker delta.

We can indeed find such primes by using the Chebotarev density theorem. (After taking $m_{1}, \cdots, m_{k}$ satisfying (A2) and (A3), take $\ell_{1} \nmid n$ such that the fixed field of the Frobenius element at $\ell_{1}$ in $\operatorname{Gal}\left(K\left(\sqrt{-1}, \sqrt{m_{1}}, \cdots, \sqrt{m_{k}}\right) / \mathbb{Q}\right)$ is $\mathbb{Q}\left(\sqrt{-1}, \sqrt{m_{2}}, \cdots\right.$, $\sqrt{m_{k}}$ ), and so on.) By Lemma 5.2 below, which is proved by using a result in [13], we can take odd positive integers $s$ and $t$ such that

$$
s \ell_{1} \cdots \ell_{k}-16 t m_{1}^{n} \cdots m_{k}^{n}=1
$$

and $s t$ has at most 5 prime factors.

Lemma 5.2. Let $a$ and $b$ be nonzero coprime integers. If $a b$ is even and negative, then there exist odd positive integers $c$ and $d$ such that $a c+b d=1$ and $c d$ has at most 5 prime factors.

Proof. We may assume $a$ is negative. Take odd integers $c_{0}$ and $d_{0}$ satisfying $a c_{0}+b d_{0}=$ 1 and consider the polynomial $F(x):=\left(2 a x-d_{0}\right)\left(2 b x+c_{0}\right) \in \mathbb{Z}[x]$. By assumption, we have $8 a b\left(a c_{0}+b d_{0}\right) \neq 0$. Moreover, for any prime $p$, there is an integer $e$ such that $F(e) \not \equiv 0(\bmod p)$. Then there exist infinitely many positive integers $e^{\prime}$ such that $F\left(e^{\prime}\right)$ has at most 5 prime factors (cf. [13, Chapter 10], [10]). Take such an $e^{\prime}$ so that both $c=c_{0}+2 b e^{\prime}$ and $d=d_{0}-2 a e^{\prime}$ are positive. These $c$ and $d$ satisfy the assertion of this lemma.

Put $l=s \ell_{1} \cdots \ell_{k}$ and $m=t m_{1}^{n} \cdots m_{k}^{n}$. Let $A$ be the elliptic curve defined by the Weierstrass equation

$$
y^{2}+x y=x^{3}+8 m x^{2}+\operatorname{lm} x .
$$

The discriminant $\Delta_{A}$ of this curve is $\Delta_{A}=l^{2} m^{2}=m^{2}(16 m+1)^{2}$. As shown in [18, Lemma 1], $A$ is semistable and $A[2] \subset A(\mathbb{Q})$. In fact, the points $P_{1}=(0,0), P_{2}=$ $(-4 m, 2 m)$ and $P_{3}=\left(-\frac{l}{4}, \frac{l}{8}\right)$ have order 2. Furthermore, $A$ has split multiplicative reduction at $\ell_{1}, \cdots, \ell_{k}, m_{1}, \cdots, m_{k}$ (cf. [18, p. 383]). We have an isomorphism

$$
\lambda_{K}: H^{1}(K, A[2]) \stackrel{\sim}{\longrightarrow} \mathcal{K}=\left\{(x, y, z) \in\left(K^{\times} / K^{\times 2}\right)^{\oplus 3} \mid x y z=1\right\}
$$

such that the image of a point $P \in A(K) \backslash A(K)[2]$ under the composite map

$$
A(K) \longrightarrow A(K) / 2 A(K) \hookrightarrow H^{1}(K, A[2]) \stackrel{\lambda_{K}}{\longrightarrow} \mathcal{K}
$$

is $\left(x(P), x(P)+4 m, x(P)+\frac{l}{4}\right.$ ), where $x(P)$ is the $x$-coordinate of $P$ (cf. [18, Section 3]). Moreover, if we define the subgroup $\mathcal{K}_{v}$ of $\left(K_{v}^{\times} / K_{v}^{\times 2}\right)^{\oplus 3}$ for a prime $v$ of $K$ similarly, then there is an isomorphism $H^{1}\left(K_{v}, A[2]\right) \stackrel{\sim}{\longrightarrow} \mathcal{K}_{v}$ compatible with $\lambda_{K}$, and the image of $A\left(K_{v}\right) / 2 A\left(K_{v}\right)$ in $\mathcal{K}_{v}$ has been described explicitly (cf. [3] and [18, 
Lemma 2]). For instance, if $A$ has good reduction at a non-archimedean prime $v$ not above 2 , then the image of $A\left(K_{v}\right) / 2 A\left(K_{v}\right)$ is the subgroup of $\mathcal{K}_{v}$ generated by units of $K_{v}$. In particular, the image of the 2-Selmer group $\operatorname{Sel}_{2}(A / K)$ under $\lambda_{K}$ is contained in

$$
\mathcal{K}_{\Sigma}:=\left\{(x, y, z) \in \mathcal{K} \mid \overline{\operatorname{ord}_{v}}(x)=\overline{\operatorname{ord}_{v}}(y)=0 \text { for any } v \notin \Sigma\right\},
$$

where $\Sigma$ is the set of primes of $K$ consisting of the archimedean primes and the primes dividing $2 l m$, and $\overline{\operatorname{ord}_{v}}: K_{v}^{\times} / K_{v}^{\times 2} \rightarrow \mathbb{Z} / 2 \mathbb{Z}$ is the homomorphism induced by the normalized valuation.

Let $\mathcal{L}$ be the subgroup of $\mathcal{K}_{\Sigma}$ generated by the classes of the elements $(q, q, 1)$ and $(q, 1, q)$ for all $q \in\left\{\ell_{1}, \cdots, \ell_{k}, m_{1}, \cdots, m_{k}\right\}$. We have $\operatorname{dim}_{\mathbb{F}_{2}} \mathcal{L}=4 k$.

Lemma 5.3. Let $h$ denote the 2 -rank of the $\Sigma$-ideal class group $\mathrm{Cl}_{\Sigma}(K)$ of $K$. Then we have $\operatorname{dim}_{\mathbb{F}_{2}} \mathcal{K}_{\Sigma} / \mathcal{L} \leq 14 n+2 h$.

Proof. We have an exact sequence

$$
1 \longrightarrow\left(\mathcal{O}_{\Sigma}^{\times} / \mathcal{O}_{\Sigma}^{\times 2}\right)^{\oplus 2} \longrightarrow \mathcal{K}_{\Sigma} \longrightarrow\left(\mathrm{Cl}_{\Sigma}(K)[2]\right)^{\oplus 2} \longrightarrow 1
$$

where $\mathcal{O}_{\Sigma}^{\times}$is the group of $\Sigma$-units of $K$. Since $K$ is a totally real field of degree $n$, there exist exactly $n$ archimedean primes. Since $2 s t$ has at most 6 prime factors, the number of non-archimedean primes in $\Sigma$ is at most $6 n+2 k$ by (A3). Hence we have $\operatorname{dim}_{\mathbb{F}_{2}} \mathcal{O}_{\Sigma}^{\times} / \mathcal{O}_{\Sigma}^{\times 2} \leq 7 n+2 k$. This implies

$$
\operatorname{dim}_{\mathbb{F}_{2}} \mathcal{K}_{\Sigma}-\operatorname{dim}_{\mathbb{F}_{2}} \mathcal{L} \leq 2(7 n+2 k)+2 h-4 k=14 n+2 h
$$

as desired.

The following proposition is proved by an argument given in [18, Section 2].

Proposition 5.4. $\mathcal{L} \cap \lambda_{K}\left(\operatorname{Sel}_{2}(A / K)\right)=\{1\}$.

Proof. Take an element $(x, y, z) \in \mathcal{L} \cap \lambda_{K}\left(\operatorname{Sel}_{2}(A / K)\right)$ and suppose $y$ is represented by $q:=\ell_{1}^{e_{1}} \cdots \ell_{k}^{e_{k}} m_{1}^{f_{1}} \cdots m_{k}^{f_{k}}\left(e_{i}, f_{j} \in\{0,1\}\right)$. It is known that $y$ is contained in the kernel of the natural map $K^{\times} / K^{\times 2} \rightarrow K_{\ell_{i}}^{\times} / K_{\ell_{i}}^{\times^{2}}$ for any $i$ (cf. [3, Section 4], [18, Section 2]). This implies that $\overline{\operatorname{ord}_{\ell_{i}}}(y)=0$, i.e., $e_{i}=0$. Moreover, we have $f_{i}=0$ since $m_{i} \notin K_{\ell_{i}}^{\times^{2}}$ and $m_{j} \in K_{\ell_{i}}^{\times^{2}}$ for any $j \neq i$ by (A4). (Recall that $n=[K: \mathbb{Q}]$ is odd.) Thus, $y$ is trivial in $K^{\times} / K^{\times 2}$. Similar argument shows that $z$ is trivial since the image of $z$ in $K_{m_{j}}^{\times} / K_{m_{j}}^{\times}{ }^{2}$ should be trivial for any $j$ and $\left(\frac{\ell_{i}}{m_{j}}\right)=(-1)^{\delta_{i, j}}$ by (A1) and (A4). This proves the assertion.

By this proposition, $\operatorname{Sel}_{2}(A / K)$ can be regarded as a subgroup of $\mathcal{K}_{\Sigma} / \mathcal{L}$. We obtain the following upper bound of the Mordell-Weil rank of $A$ over $K$.

Corollary 5.5. $\operatorname{rank}_{\mathbb{Z}} A(K) \leq 14 n+2 h-2$.

Proof. By Lemma 5.3 and Proposition 5.4, we have $\operatorname{dim}_{\mathbb{F}_{2}} \operatorname{Sel}_{2}(A / K) \leq 14 n+2 h$. The assertion follows from the exact sequence (1) and the fact $\operatorname{dim}_{\mathbb{F}_{2}} A(K)[2]=2$.

Combining this with Proposition 4.3, we have the following lower bound of the $n$-rank of the Tate-Shafarevich group of $A$ over $K$. 
Corollary 5.6. We have $\operatorname{rk}_{n} \amalg(A / K)[n] \geq k-14 n-2 h-8$.

Proof. If $m_{j}$ does not divide st, then the Tamagawa factor of $A$ at $m_{j}$ is equal to $2 n$, i.e., $m_{j} \in T_{A, K}$. Since $A(\mathbb{Q})[n]=0$ by $\left[18\right.$, Lemma 3], we have $\operatorname{rk}_{n}\left(\operatorname{Sel}_{n}(A / K)\right) \geq$ $t_{A, K} \geq k-5$ by Proposition 4.3. Since $A(K) / n A(K)$ is isomorphic to a direct sum of $(\mathbb{Z} / n \mathbb{Z})^{\oplus \operatorname{rank}_{\mathbb{Z}} A(K)}$ and a cyclic group of order dividing $n$, the assertion follows from Corollary 5.5 and the exact sequence (1).

Although Corollary 5.6 is sufficient for proving Theorem A for odd primes $p$, in order to complete the proof of Theorem 5.1, we show that the 2-rank of the TateShafarevich group over $K$ also becomes large if we replace the curve $A$ with its 2isogenous curve $B$ below as in [18].

Let $B$ be the elliptic curve over $\mathbb{Q}$ defined by the equation

$$
y^{2}+x y=x^{3}-16 m x^{2}-8 m x-m .
$$

The discriminant $\Delta_{B}$ of this curve is $l m$ and there exists an isogeny $f: A \rightarrow B$ of degree 2 defined over $\mathbb{Q}$. The following lower bound on the 2-rank of $\amalg(B / K)[2]$ is enough to prove Theorem 5.1.

Proposition 5.7. We have $\operatorname{dim}_{\mathbb{F}_{2}} \amalg(B / K)[2] \geq 2 k-17$.

Remark. We give here a proof based on a result of Cassels [6] as in [16]. One can also obtain a similar lower bound by the same argument as given in Kramer's paper [18].

Proof. Since $n=[K: \mathbb{Q}]$ is odd, the kernel of the restriction map $\amalg(B / \mathbb{Q}) \rightarrow$ $\amalg(B / K)$ has no element of order 2 . Hence we have only to show $\operatorname{dim}_{\mathbb{F}_{2}} \amalg(B / \mathbb{Q})[2] \geq$ $2 k-17$. Let $g: B \rightarrow A$ be the dual isogeny of $f$. We have the following relation between the Selmer groups $\operatorname{Sel}_{f}(A / \mathbb{Q})$ and $\operatorname{Sel}_{g}(B / \mathbb{Q})$ associated with the isogenies $f$ and $g$ (cf. [16, Theorem 1]):

$$
\operatorname{dim}_{\mathbb{F}_{2}} \operatorname{Sel}_{g}(B / \mathbb{Q}) \geq \operatorname{dim}_{\mathbb{F}_{2}} \operatorname{Sel}_{f}(A / \mathbb{Q})+\sum_{q}\left(u_{A, q}-u_{B, q}\right)-1 .
$$

Here $q$ runs over all prime numbers at which $A$ and $B$ have bad reduction and we denote by $u_{A, q}$ and $u_{B, q}$ the normalized 2-adic valuations of the Tamagawa factors of $A$ and $B$ at $q$. Since $A$ and $B$ are semistable and $\Delta_{A}=\Delta_{B}^{2}$, we have $u_{A, q} \geq u_{B, q}$ for any prime $q$ at which $A$ and $B$ have bad reduction. Moreover, we have $u_{A, q}-u_{B, q}=1$ if $q$ is one of the primes $\ell_{1}, \cdots, \ell_{k}, m_{1}, \cdots, m_{k}$ since both $A$ and $B$ have split multiplicative reduction at $q$. Hence we have

$$
\operatorname{dim}_{\mathbb{F}_{2}} \operatorname{Sel}_{g}(B / \mathbb{Q}) \geq \operatorname{dim}_{\mathbb{F}_{2}} \operatorname{Sel}_{f}(A / \mathbb{Q})+2 k-1 \geq 2 k-1 .
$$

By the exact sequence

$$
B(\mathbb{Q})[2] \longrightarrow A(\mathbb{Q})[f] \longrightarrow \operatorname{Sel}_{g}(B / \mathbb{Q}) \longrightarrow \operatorname{Sel}_{2}(B / \mathbb{Q})
$$

(cf. [16, Proposition 1]), we have $\operatorname{dim}_{\mathbb{F}_{2}} \operatorname{Sel}_{2}(B / \mathbb{Q}) \geq \operatorname{dim}_{\mathbb{F}_{2}} \operatorname{Sel}_{g}(B / \mathbb{Q})-1 \geq 2 k-2$. By the same argument as in the proof of Proposition 5.4 and Corollary 5.5, we have $\operatorname{rank}_{\mathbb{Z}} B(\mathbb{Q})=\operatorname{rank}_{\mathbb{Z}} A(\mathbb{Q}) \leq 14$ (see also the proof of Corollary 6.2). Therefore, we have $\operatorname{dim}_{\mathbb{F}_{2}} \amalg(B / \mathbb{Q})[2] \geq 2 k-2-14-1=2 k-17$ by (1) and the fact that $B(\mathbb{Q})[2] \cong \mathbb{Z} / 2 \mathbb{Z}$. 
The isogeny $f: A \rightarrow B$ induces an isomorphism $\amalg(A / K)[n] \cong \amalg(B / K)[n]$ since the degree of $f$ is prime to $n$. Hence we have

$$
\operatorname{rk}_{2 n} \amalg(B / K) \geq k-14 n-2 h-8
$$

by Corollary 5.6 and Proposition 5.7. Thus the elliptic curve $E=B$ with $k=$ $\kappa+14 n+2 h+8$ satisfies the assertion of Theorem 5.1.

\section{The case $p=2$}

In this section, we complete the proof of Theorem $\mathrm{A}$ for $p=2$. The proof is obtained by combining Proposition 4.3 with a result of Hoffstein-Luo [14], a variant of Waldspurger's result on the behavior of central values of the Hasse-Weil $L$-functions under quadratic twists.

Let $K$ be a quadratic field with fundamental discriminant $D$. For an arbitrary positive integer $k$, take distinct odd primes $\ell_{1}, \cdots, \ell_{k}, m_{1}, \cdots, m_{k}$ satisfying the conditions (A1), (A3) and (A4) in the preceding section. (We can indeed take such primes by the Chebotarev density theorem; $\ell_{1}$ is taken so that the fixed field of the Frobenius element in $\operatorname{Gal}\left(\mathbb{Q}\left(\sqrt{-1}, \sqrt{D}, \sqrt{m_{1}}, \cdots, \sqrt{m_{k}}\right) / \mathbb{Q}\right)$ is $\mathbb{Q}\left(\sqrt{-1}, \sqrt{D m_{1}}, \sqrt{m_{2}}, \cdots, \sqrt{m_{k}}\right)$. Then, by Lemma 5.2, there exist odd positive integers $s$ and $t$ such that $s \ell_{1} \cdots \ell_{k}-$ $16 t m_{1} \cdots m_{k}=1$ and $s t$ has at most 5 prime factors. Let $A$ be an elliptic curve defined by the equation (5) with $l=s \ell_{1} \cdots \ell_{k}$ and $m=t m_{1} \cdots m_{k}$ (not same as in the preceding section). The following proposition is proved by using a result of [14]. We denote by $E_{a}$ the quadratic twist of an elliptic curve $E$ over $\mathbb{Q}$ corresponding to a quadratic extension $\mathbb{Q}(\sqrt{a}) / \mathbb{Q}$.

Proposition 6.1. There exists a square-free integer $d$ with at most 4 prime factors such that $\operatorname{rank}_{\mathbb{Z}} A_{d}(K)=\operatorname{rank}_{\mathbb{Z}} A_{d}(\mathbb{Q}), d \equiv 1(\bmod 8)$, and $\left(\frac{d}{q}\right)=1$ for any prime $q$ dividing Dlm.

Proof. Let $S$ be the set of prime numbers dividing $2 \mathrm{Dlm}$. By applying [14, Theorem] to $A_{D}$ and $S$, we obtain an integer $d$ with at most 4 prime factors which satisfies $L\left(A_{D d}, 1\right) \neq 0$ and $\left(\frac{d}{q}\right)=1$ for any $q \in S$. Here $L\left(A_{D d}, s\right)$ is the Hasse-Weil $L$-function of $A_{D d}$. By a result of Kolyvagin on the Birch and Swinnerton-Dyer conjecture $([17])$, we have $\operatorname{rank}_{\mathbb{Z}} A_{D d}(\mathbb{Q})=0$. This implies

$$
\operatorname{rank}_{\mathbb{Z}} A_{d}(K)=\operatorname{rank}_{\mathbb{Z}} A_{d}(\mathbb{Q})+\operatorname{rank}_{\mathbb{Z}} A_{D d}(\mathbb{Q})=\operatorname{rank}_{\mathbb{Z}} A_{d}(\mathbb{Q})
$$

as desired.

By the argument of Kramer [18] used in the preceding section, we obtain the following upper bound of the Mordell-Weil rank of $A_{d}$ over $K$.

Corollary 6.2. We have $\operatorname{rank}_{\mathbb{Z}} A_{d}(K)=\operatorname{rank}_{\mathbb{Z}} A_{d}(\mathbb{Q}) \leq 20$.

Proof. If we put $d=4 e+1$, then $A_{d}$ has a Weierstrass equation

$$
y^{2}+x y=x^{3}+(8 m d+e) x^{2}+l m d^{2} x .
$$

The discriminant of this Weierstrass model is $l^{2} m^{2} d^{6}$ and $A_{d}(\mathbb{Q})$ contains $A_{d}[2]$. As in the preceding section, $\operatorname{Sel}_{2}\left(A_{d} / \mathbb{Q}\right)$ is regarded as a subgroup of

$$
\mathcal{Q}_{\Sigma}=\left\{(x, y, z) \in\left(\mathbb{Q}^{\times} / \mathbb{Q}^{\times 2}\right)^{\oplus 3} \mid x y z=1, \overline{\operatorname{ord}_{q}}(x)=\overline{\operatorname{ord}_{q}}(y)=0 \text { for any } q \notin \Sigma\right\},
$$


where $\Sigma$ is the set of prime numbers dividing $2 \mathrm{dlm}$. Moreover, any nonzero element of $\operatorname{Sel}_{2}\left(A_{d} / \mathbb{Q}\right)$ is not contained in the subgroup of $\mathcal{Q}_{\Sigma}$ generated by the classes of $(q, q, 1)$ and $(q, 1, q)$ for all $q \in\left\{\ell_{1}, \cdots, \ell_{k}, m_{1}, \cdots, m_{k}\right\}$ since the assumption $\left(\frac{d}{q}\right)=1$ implies the local condition at $q$ for defining the 2-Selmer group does not change by the quadratic twist corresponding to $\mathbb{Q}(\sqrt{d})$ (see the proof of Proposition 5.4). Hence we have

$$
\operatorname{dim}_{\mathbb{F}_{2}} \operatorname{Sel}_{2}\left(A_{d} / \mathbb{Q}\right) \leq \operatorname{dim}_{\mathbb{F}_{2}} \mathcal{Q}_{\Sigma}-4 k=2(2 k+5+4+2)-4 k=22 .
$$

This implies $\operatorname{rank}_{\mathbb{Z}} A_{d}(\mathbb{Q}) \leq \operatorname{dim}_{\mathbb{F}_{2}} \operatorname{Sel}_{2}\left(A_{d} / \mathbb{Q}\right)-\operatorname{dim}_{\mathbb{F}_{2}} A_{d}(\mathbb{Q})[2] \leq 20$, as desired.

Corollary 6.3. We have $\operatorname{dim}_{\mathbb{F}_{2}} \amalg\left(A_{d} / K\right)[2] \geq 2 k-31$.

Proof. Since $A_{d}$ has split multiplicative reduction with even Tamagawa factor at each $q \in\left\{\ell_{1}, \cdots, \ell_{k}, m_{1}, \cdots, m_{k}\right\}$ not dividing st and any such $q$ remains prime in $K$, we have $t_{A_{d}, K} \geq 2 k-5$. By Proposition 4.3, we have $\operatorname{dim}_{\mathbb{F}_{2}} \operatorname{Sel}_{2}\left(A_{d} / K\right) \geq 2 k-9$. Hence we have $\operatorname{dim}_{\mathbb{F}_{2}} \amalg\left(A_{d} / K\right)[2] \geq 2 k-9-\operatorname{dim}_{\mathbb{F}_{2}} A_{d}(K) / 2 A_{d}(K) \geq 2 k-31$ by (1) and Corollary 6.2.

By taking $k$ large arbitrarily, this corollary implies that the 2-rank of $\amalg\left(A_{d} / K\right)[2]$ is unbounded as $d$ varies. The proof of Theorem A has been completed.

We can also give a proof of Theorem $\mathrm{A}$ for $p=2$ by considering the 2-rank of $\amalg\left(B_{d} / K\right)$ instead of $\amalg\left(A_{d} / K\right)$. As in the preceding section, we can show that

$$
\begin{aligned}
\operatorname{dim}_{\mathbb{F}_{2}} \amalg\left(B_{d} / \mathbb{Q}\right)[2] & =\operatorname{dim}_{\mathbb{F}_{2}} \operatorname{Sel}_{2}\left(B_{d} / \mathbb{Q}\right)-\operatorname{rank}_{\mathbb{Z}} B_{d}(\mathbb{Q})-\operatorname{dim}_{\mathbb{F}_{2}} B_{d}(\mathbb{Q})[2] \\
& \geq(2 k-8-1)-20-1=2 k-30
\end{aligned}
$$

by using [16, Theorem 1] and Corollary 6.2. (Recall that $B_{d}$ is isogenous to $A_{d}$ and $B_{d}$ has semistable reduction at any prime not dividing $d$.) As we remarked before, this does not imply the assertion of Theorem A immediately since $\operatorname{Ker}\left(\amalg\left(B_{d} / \mathbb{Q}\right) \rightarrow\right.$ $\left.\amalg\left(B_{d} / K\right)\right)$ may have a large subgroup of exponent 2 in general. However, we can apply the following lemma in this case.

Lemma 6.4. Let $F^{\prime} / F$ be a Galois extension of number fields such that $\left[F^{\prime}: F\right]$ is a prime $p$. For any elliptic curve $E$ defined over $F$ satisfying $\operatorname{rank}_{\mathbb{Z}} E\left(F^{\prime}\right)=\operatorname{rank}_{\mathbb{Z}} E(F)$, we have

$$
\operatorname{dim}_{\mathbb{F}_{p}} \amalg\left(E / F^{\prime}\right)[p] \geq \operatorname{dim}_{\mathbb{F}_{p}} \amalg(E / F)[p]-2 .
$$

Proof. By the inflation-restriction sequence, the kernel of the restriction map $\amalg(E / F) \rightarrow \amalg\left(E / F^{\prime}\right)$ is regarded as a subgroup of $H^{1}\left(G, E\left(F^{\prime}\right)\right)$, where $G=$ $\operatorname{Gal}\left(F^{\prime} / F\right)$. We have only to prove that the $p$-rank of $H^{1}\left(G, E\left(F^{\prime}\right)\right)$ is at most 2. If we denote by $T$ the torsion subgroup of $E\left(F^{\prime}\right)$, then $G$ acts trivially on the free $\mathbb{Z}$-module $E\left(F^{\prime}\right) / T$. Indeed, $P^{\sigma}-P$ is contained in $T$ for any $P \in E\left(F^{\prime}\right)$ and any $\sigma \in G$ by the assumption $\operatorname{rank}_{\mathbb{Z}} E\left(F^{\prime}\right)=\operatorname{rank}_{\mathbb{Z}} E(F)$. Hence we have $H^{1}\left(G, E\left(F^{\prime}\right) / T\right)=\operatorname{Hom}\left(G, E\left(F^{\prime}\right) / T\right)=0$. On the other hand, $H^{1}(G, T)$ is of exponent $p$ and its $p$-rank is not greater than $\operatorname{dim}_{\mathbb{F}_{p}} T[p] \leq 2$. The claim is proved.

Since $\operatorname{rank}_{\mathbb{Z}} B_{d}(\mathbb{Q})=\operatorname{rank}_{\mathbb{Z}} B_{d}(K)$ by Proposition 6.1, we have $\operatorname{dim}_{\mathbb{F}_{2}}$ $\amalg\left(B_{d} / K\right)[2] \geq 2 k-32$. This implies the assertion of Theorem A for $p=2$. 


\section{Acknowledgements}

The author would like to thank Yoshitaka Hachimori for helpful discussions, and the referee for valuable suggestions. The author was partially supported by KAKENHI (Grant-in-Aid for Young Scientists (B)) 18740013.

\section{References}

[1] N. Aoki, On the Tate-Shafarevich group of semistable elliptic curves with a rational 3-torsion, Acta Arith. 112 (2004), 209-227.

[2] R. Bölling, Die Ordnung der Schafarewitsch-Tate-Gruppe kann beliebig groß werden, Math. Nachr. 67 (1975), 157-179.

[3] A. Brumer and K. Kramer, The rank of elliptic curves, Duke Math. J. 44 (1977), 715-743.

[4] D. Bump, S. Friedberg and J. Hoffstein, Nonvanishing theorems for L-functions of modular forms and their derivatives, Invent. math. 102 (1990), 543-618.

[5] J. W. S. Cassels, Arithmetic on curves of genus 1, VI. The Tate-Šafarevič group can be arbitrarily large, J. reine angew. Math. 214/215 (1964), 65-70.

[6] J. W. S. Cassels, Arithmetic on curves of genus 1, VIII. On conjectures of Birch and Swinnerton-Dyer, J. reine angew. Math. 217 (1965), 180-199.

[7] P. L. Clark and S. Sharif, Period, index and potential Ш, preprint, 2006.

[8] J. Coates and R. Sujatha, "Galois Cohomology of Elliptic Curves", Tata Institute of Fundamental Research, Narosa Publ. House, 2000.

[9] J. E. Cremona, "Algorithms for Modular Elliptic Curves", 2nd edition, Cambridge University Press, 1997.

[10] H. Diamond and H. Halberstam, Some applications of sieves of dimension exceeding 1, in "Sieve Methods, Exponential Sums, and their Applications in Number Theory", London Math. Soc. Lecture Note Series, vol. 237, Cambridge University Press, 1997, pp. 101-107.

[11] T. Fisher, Some examples of 5 and 7 descent for elliptic curves over $\mathbb{Q}$, J. Eur. Math. Soc. 3 (2001), 169-201.

[12] R. Greenberg, Iwasawa theory for elliptic curves, in "Arithmetic Theory of Elliptic Curves", Lecture Notes in Math., vol. 1716, Springer-Verlag, 1999, pp. 51-144.

[13] H. Halberstam and H.-E. Richert, "Sieve Methods", Academic Press, 1974.

[14] J. Hoffstein and W. Luo, Nonvanishing of L-series and the combinatorial sieve, Math. Research Letters 4 (1997), 435-444.

[15] R. Kloosterman, The p-part of Shafarevich-Tate groups of elliptic curves can be arbitrarily large, J. Theorie Nombres Bordeaux 17 (2005), 787-800.

[16] R. Kloosterman and E. F. Schaefer, Selmer groups of elliptic curves that can be arbitrarily large, J. Number Theory 99 (2003), 148-163.

[17] V. A. Kolyvagin, Finiteness of $E(\mathbf{Q})$ and $\amalg(E, \mathbf{Q})$ for a subclass of Weil curves, Math. USSRIzv. 32 (1989), 523-541.

[18] K. Kramer, A family of semistable elliptic curves with large Tate-Shafarevitch groups, Proc. Amer. Math. Soc. 89 (1983), 379-386.

[19] S. Lang and J. Tate, Principal homogeneous spaces over abelian varieties, Amer. J. Math. 80 (1958), 659-684.

[20] K. Matsuno, Construction of elliptic curves with large Iwasawa $\lambda$-invariants and large TateShafarevich groups, manuscr. math. 122 (2007), 289-304.

[21] B. Mazur, Rational points of abelian varieties with values in towers of number fields, Invent. math. 18 (1972), 183-266.

[22] J. S. Milne, "Arithmetic Duality Theorems", 2nd edition, BookSurge, LLC, 2006.

[23] J. H. Silverman, "The Arithmetic of Elliptic Curves", Graduate Texts in Math., vol. 106, Springer-Verlag, 1986.

Department of Mathematics, Tsuda College, 2-1-1, Tsuda-machi, Kodaira, Tokyo 1878577, JAPAN

E-mail address: matsuno@tsuda.ac.jp 